\title{
Tunable Band Pass Filter and Phase Modulator Based on Multimode Nested Mach- Zehnder Interferometer
}

Tahreer S. Mansour Alfalah

Institute of Laser for Postgraduate Studies

Iraq

tahreeralflah@yahoo.com

Ayah T. Yahiya Al-khalefa

Institute of Laser for Postgraduate Studies

Iraq

aya.thabit.khalefa@gmail.com

ABSTRACT: This paper presents a continuously tunable optical band pass filter based on nested MZI designed by two arms multimode Machzehnder interferometer was proposed. The tunability of the filter is achieved by wavelength tuning of the multimode fiber. Tunability of the filter can be generated by applying Mechanical effect which is strain on the sensing arm of MM-MZI. It has been shown from the simulated results, it is done by Optiwave soft wear obtained that the shifted central wavelength from (1546.01) to (1546.021) $\mathrm{nm}$, full width at half maximum from (279.321) to (273.681) pm, maximum gain is $22.55 \mathrm{~dB}$ and phase shift from(0) to (6.0808) rad and in the practical results obtained to the shifted central wavelength from(1545.762) to (1545.764)nm, FWHM from (197.529) to (283081)pm, maximum gain is 21.66 dB, and phase shift from (0) to (6.059) rad. Observed from the results, the relation between the shifted central wavelength and strain is nonlinear.

Keywords: Fiber - Optic Sensors, Multimode Mach-Zehnder Interferometer (MM-MZI), Strain Measurement

DOI: $10.6025 / \mathrm{stj} / 2018 / 7 / 2 / 43-51$

Received: 10 May 2018, Revised 19 June 2018, Accepted 28 June 2018

(C) 2018 DLINE. All Rights Reserved

\section{Introduction}

We present a new tunable all-fiber compact multimode fiber (MMF)-based filter and its applications in fiber sensors. The expression of the transmission of the optical filter is the same as that of a regular Mach-Zehnder interferometer (MZI) but with an additional optical phase shift in the sinusoidal function, which makes the MMF-based filter tunable. The phase shift could be changed by properly adjusting of a mechanical effect i.e. (strain). The proposed tunable filter has been employed for intensity interrogation of a fiber Bragg grating (FBG)-based temperature sensor where the MMF-based filter serves as an band pass filter. With the tuning technique. Multimode fiber (MMF) based MZI has been proposed by our group for simultaneous measurement of strain [1]. 


\section{Theoretical Overview}

The schematic diagram of a MM-MZI is shown in figure 1. It consist of two multimode couplers the first acts as a splitter to split light in to two arms reference and sensing arms and the second acts as a combiner. When applied micro strain on the cross section area of multimode fiber on the sensing arm, resulting optical path difference between these arms. The light intensity of the output of MZI can be expressed as [2]:

$$
I=I_{r}+I_{s}+2 \sqrt{I_{r} I_{s}} \cos (\Delta \varphi)
$$

Where $I_{r}$ and $I_{s}$ are the intensities of reference and sensing arm.

The phase difference between the sensing arm and reference arm is Described by [2]:

$$
\Delta \varphi=\frac{2 \pi \Delta L n_{e f f}}{\lambda}
$$

Where $\Delta L$ is the change in optical path length, $\lambda$ is the wavelength in the vacuum, and $n_{\text {eff }}$ is the effective refractive index of the mode.

Output power of Mach-Zehnder Interferometer is described by [2]:

$$
P_{\text {out }}=P_{\text {in }} \cos ^{2}(0.5 \Delta \varphi)
$$

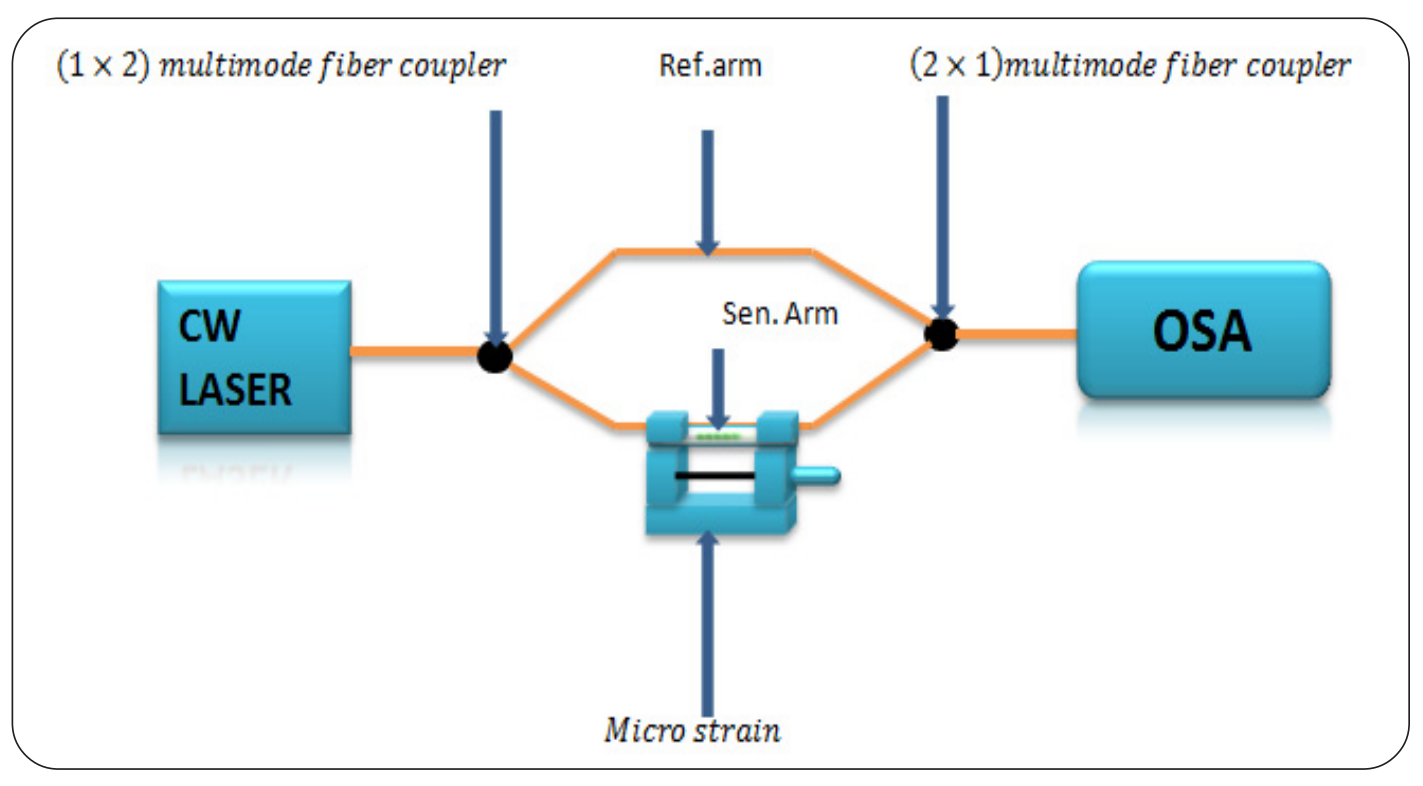

Figure 1. Schematic diagram of tunable optical fiber MM-MZI band pass filter

\section{Experiments and Setup Assembly}

\subsection{Theoretical Optiwave}

Figure (2) shows the simulation setup of the system utilizing OptiSystem software.

The system consist of continues laser source, two multimode optical couplers the first acts as a splitter and the and the other acts as a combiner, and finally connect to the Optical spectrum analyzer (OSA) to see the output signal. 


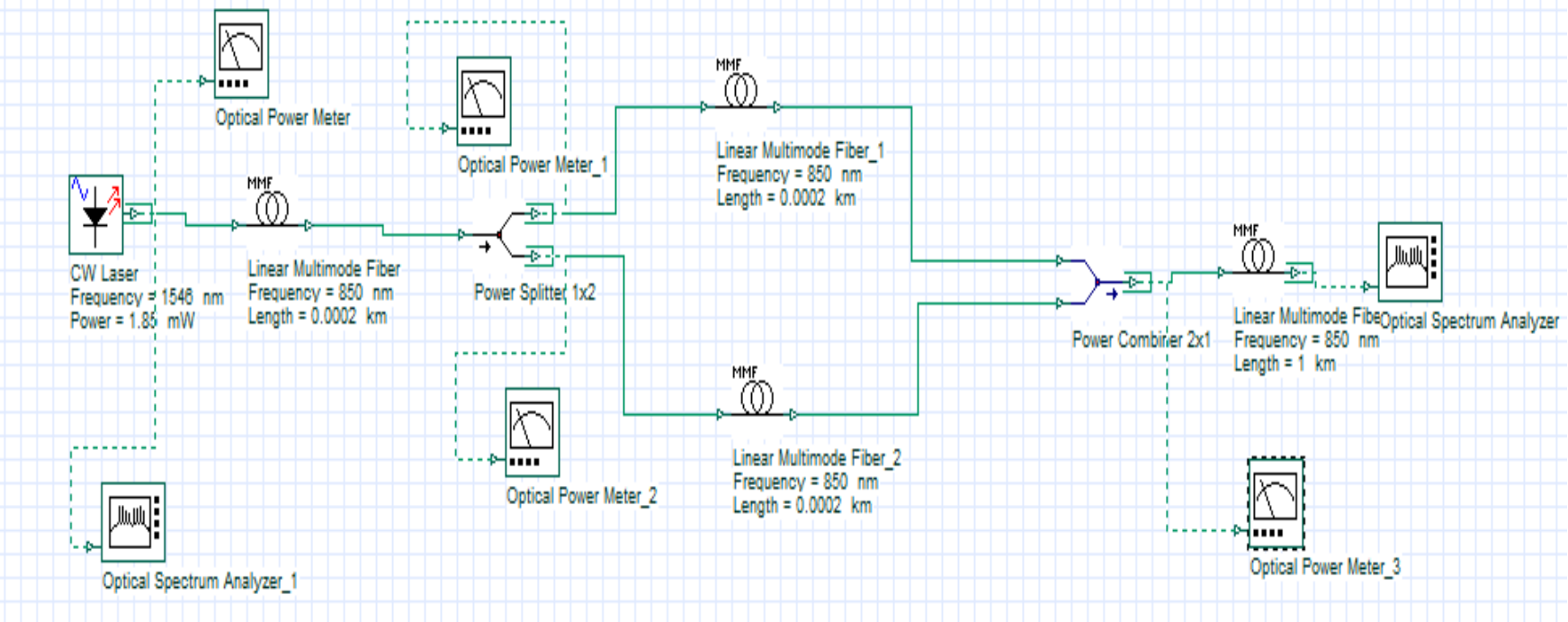

Figure 2. The designed model of simulated system with Optisystem software

Continues wave laser have specifications:

\begin{tabular}{|c|l|}
\hline Wavelength $\mathbf{n m}$ & Power $\mathbf{m W}$ \\
\hline $\mathbf{1 5 4 6}$ & 1.85 \\
\hline
\end{tabular}

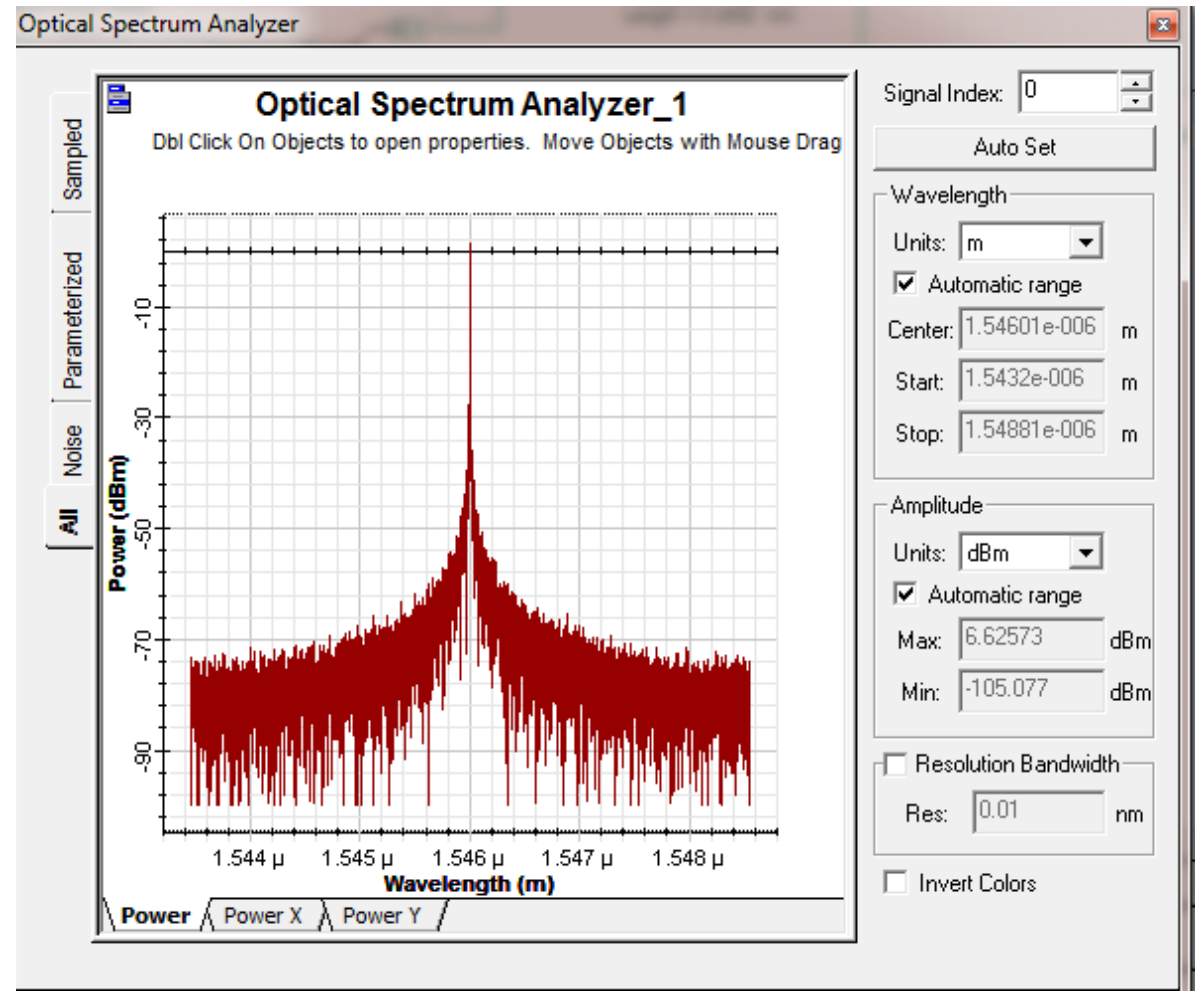

Figure 3. Signal of CW LASER source 


\subsection{Practical Setup}

Figure 4 are photographic images of the experimental parts. It comprises of continues wave laser source (CW LASER), $(1 \times 2)$ input multimode fiber coupler acts as splitter, Dual multimode fiber reference and effect a arms respectively, $(2 \times 1)$ output multimode fiber coupler acts as combiner, and optical spectrum analyzer(OSA) to visualize phase shift in the spectrum of the source by applied manual elongation measuring instrument i.e. (micro strain) on the second arm of MM-MZI.

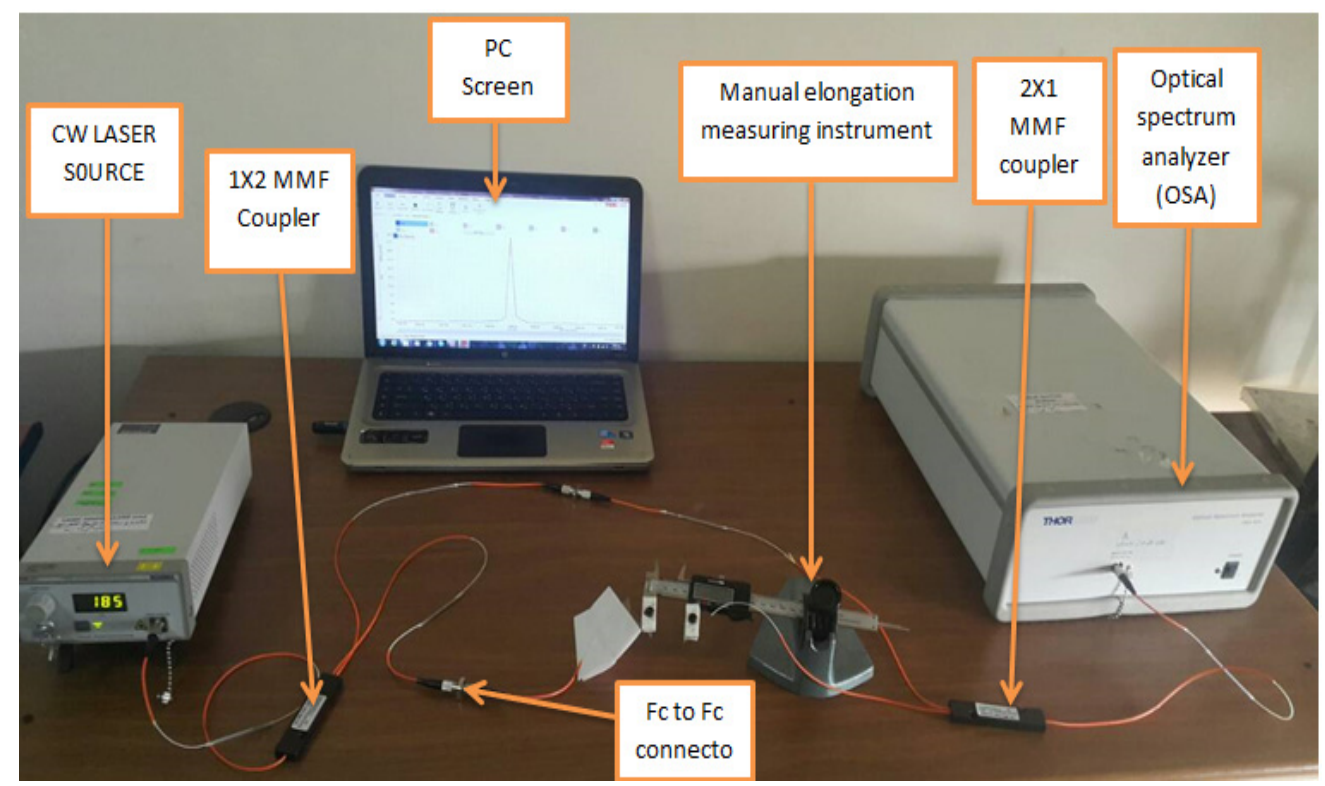

Figure 4. A photographic image for the experimental setup when applied micro strain on the cross section area of MMF

\section{Results and Discussed}

In this work, we designed and implemented a tunable optical band pass filter based on MM-MZI. the simulation and experiment work for this filter is demonstrated and constructed after designed using nested MZIs. From simulated work we obtained results such as figure 5 .

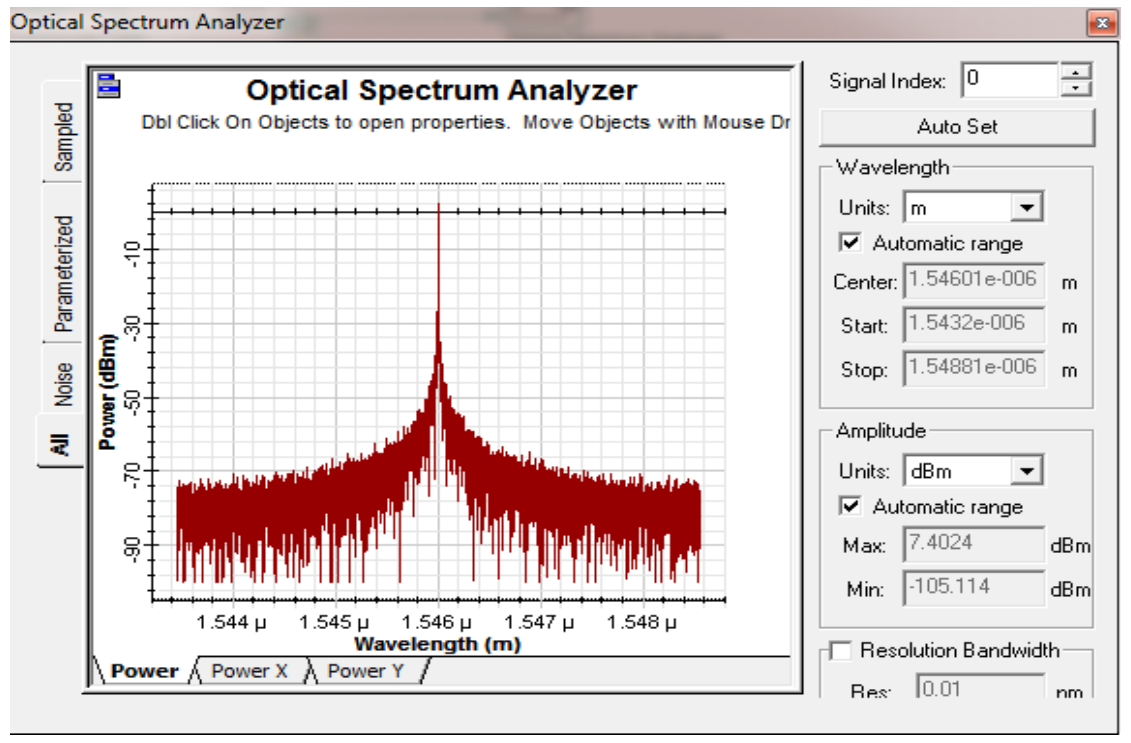

Figure (a). Signal when applied zero strain 

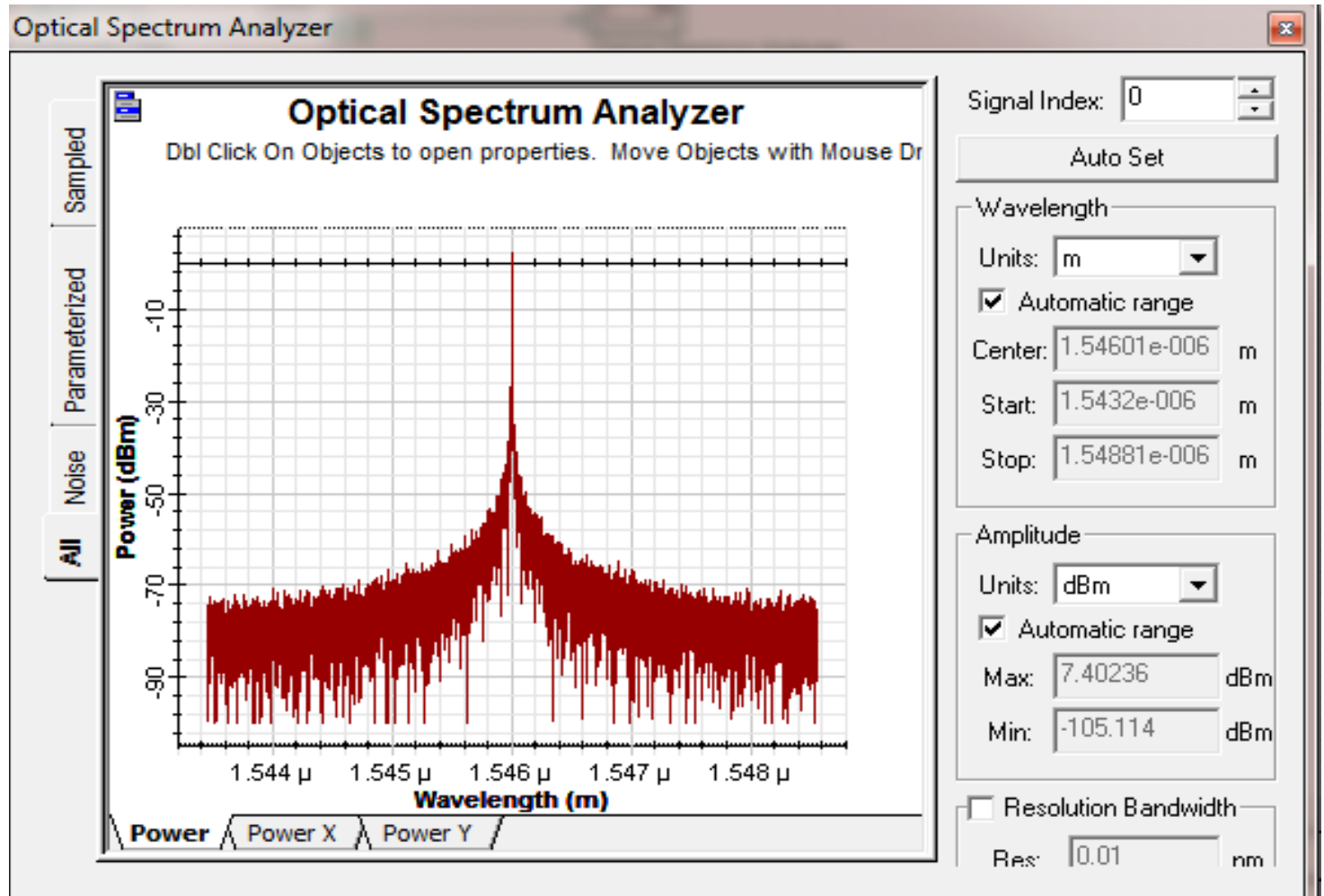

Figure (b). At strain $=0.09 \mathrm{~mm}$
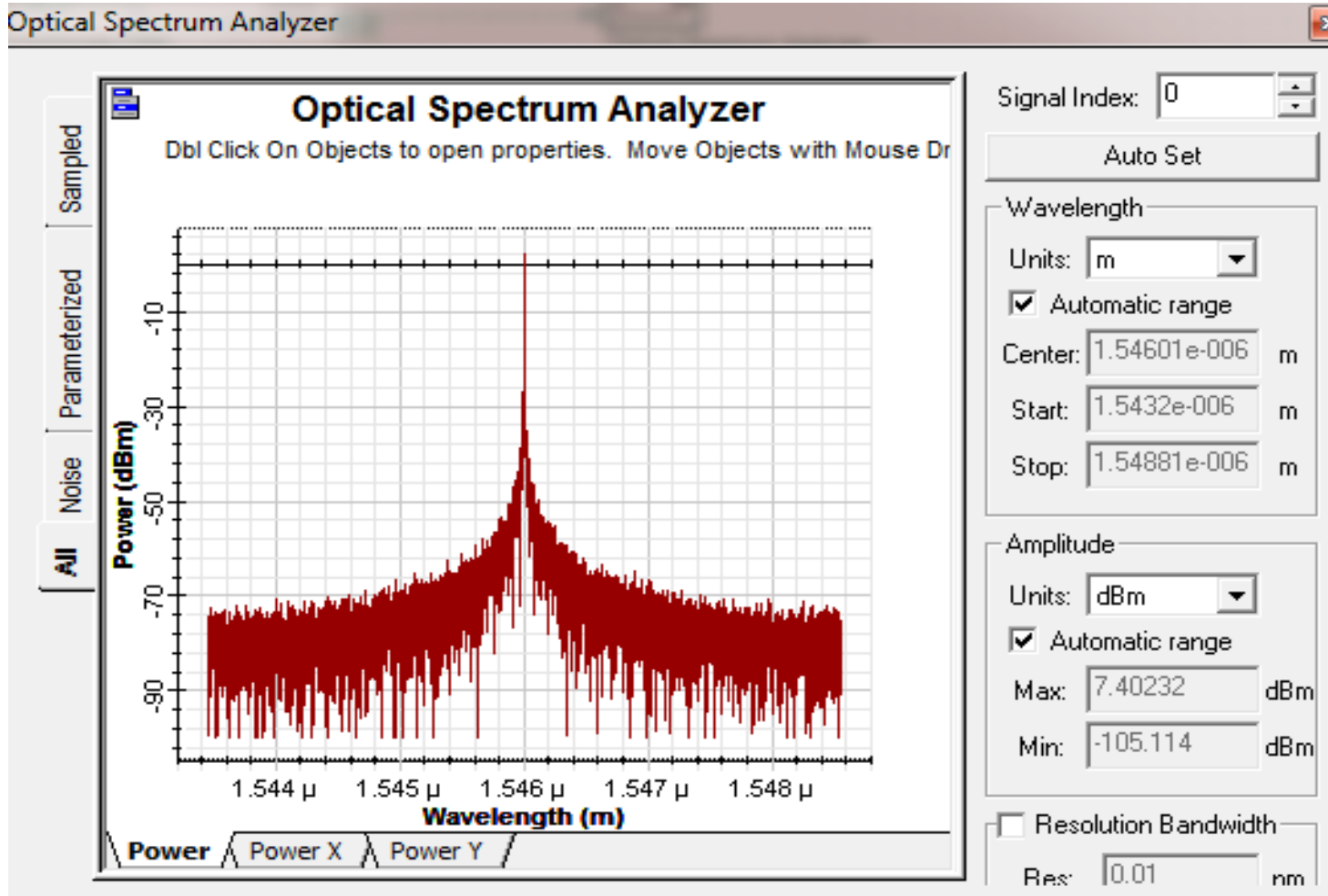

Figure $(\mathrm{c})$. At strain $=0.24 \mathrm{~mm}$ 

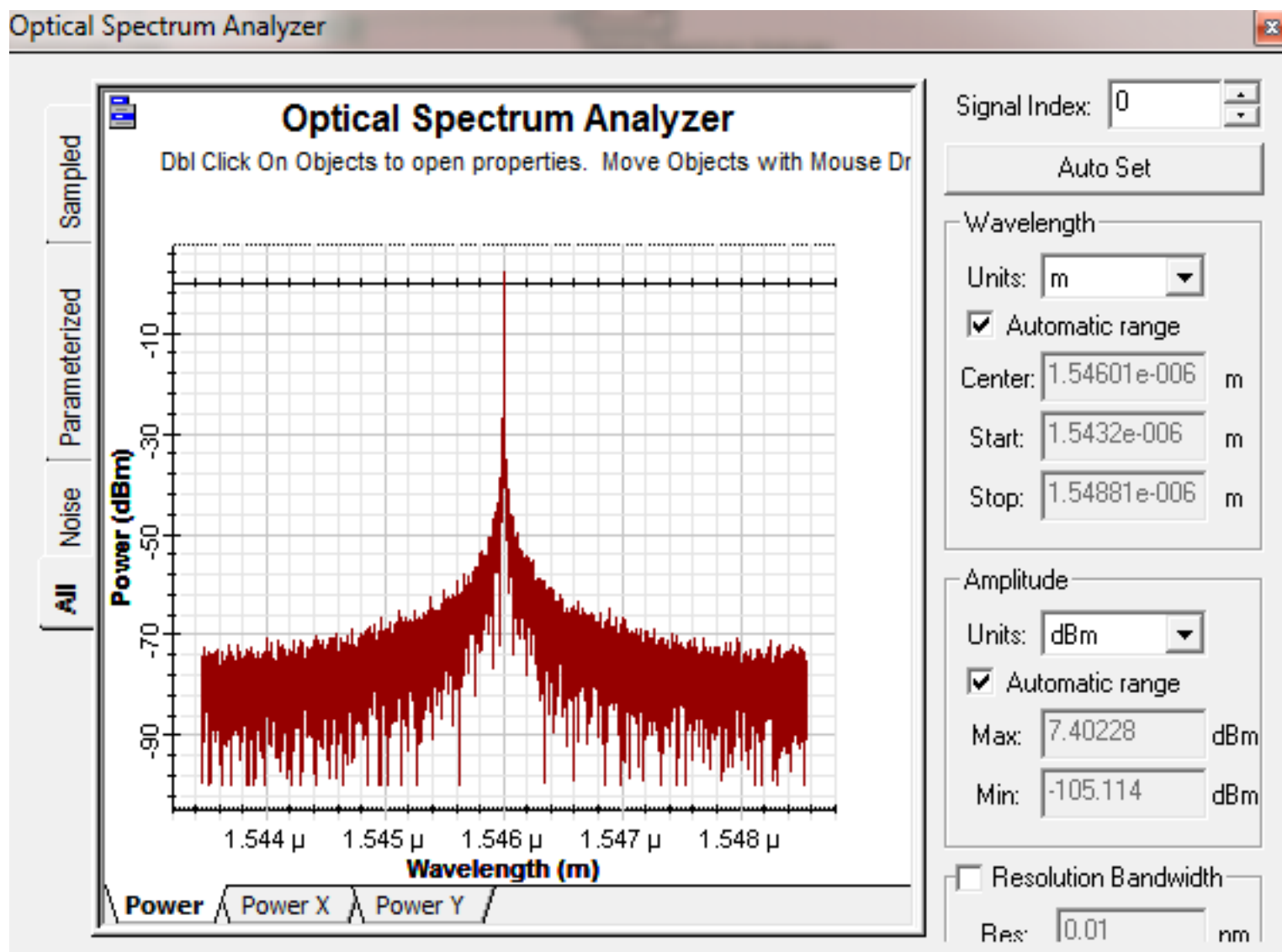

Figure (d). At strain $=0.31 \mathrm{~mm}$

Figure 5. These figures obtained from optiwave software when applied varies value of strain on the cress section area of MMF

These figures it summarizes in the table (1)

\begin{tabular}{|c|c|c|c|c|}
\hline $\operatorname{Strain}(\mathbf{m} \varepsilon)$ & Central wavelength (nm) & FWHM (pm) & $A_{d B}=20 \log P_{0}$ & $\Delta \varphi(r a d) \times 10^{-11}$ \\
\hline $\mathbf{0}$ & 1546.01 & 279.321 & 22.55 & 0 \\
\hline 0.09 & 1546.015 & 281.527 & 22.34 & 2.0269 \\
\hline 0.24 & 1546.019 & 275.762 & 21.75 & 4.0519 \\
\hline 0.31 & 1546.021 & 273.681 & 21.87 & 6.0808 \\
\hline
\end{tabular}

Table 1. Effect different values of strain on the central wavelength, FWHM, gain and phase shift

In practically, when we are applied micro strain on the second arm of MM-MZI, the central wavelength $(\lambda)$ is shifted to red shift as figure (6). The effected of micro strain on the central wavelength $(\lambda)$, full width at half maximum (FWHM), gain (A) and phase $\operatorname{shift}(\Delta \varphi)$ is listed in table (2).

\begin{tabular}{lllllll}
\hline 48 & Signals and Telecommunication Journal & Volume & 7 & Number & 2 & September \\
\hline
\end{tabular}




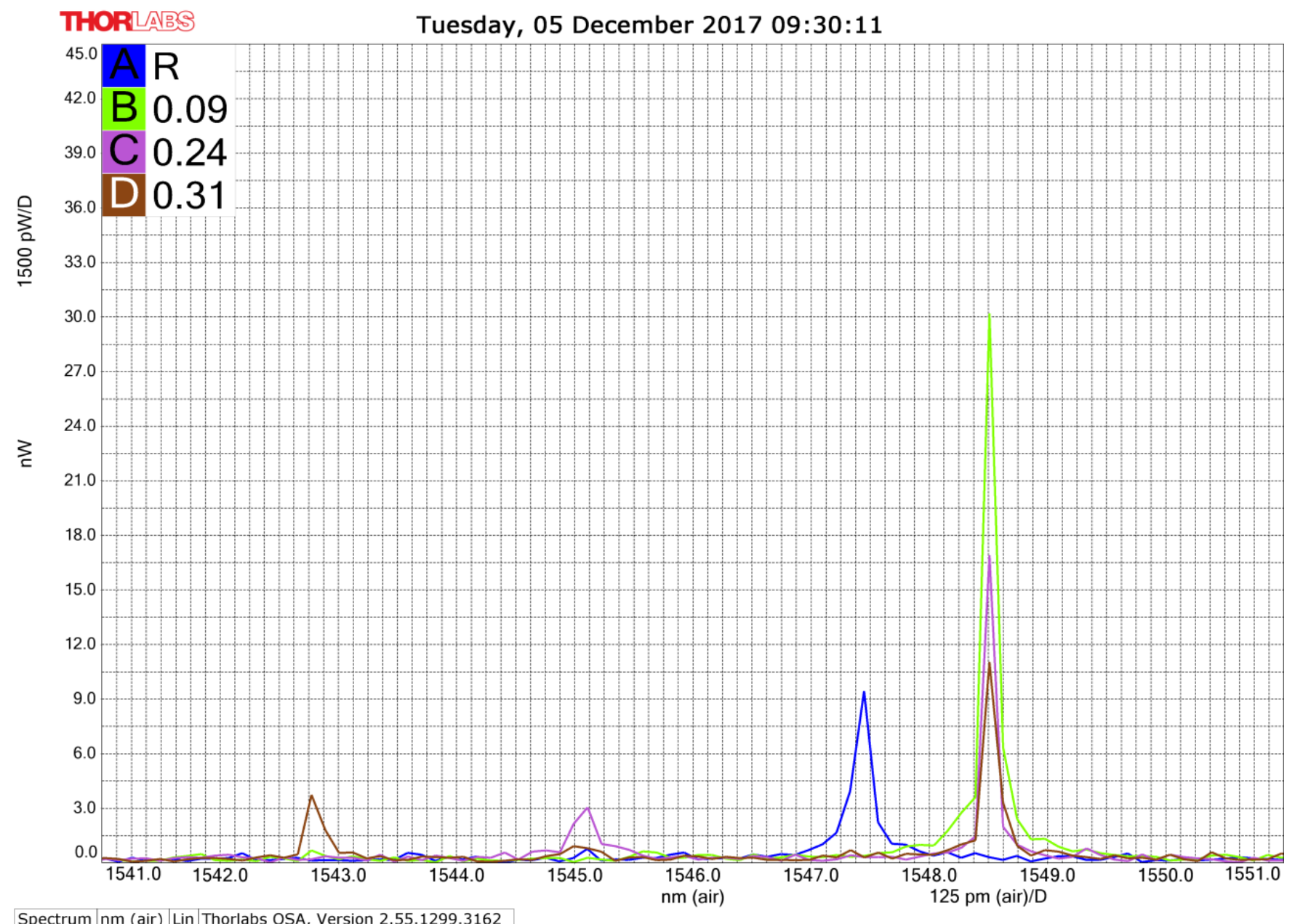

Spectrum $\mid \mathrm{nm}$ (air) $\mid$ Lin $\mid$ Thorlabs OSA, Version 2.55.1299.3162

Figure 6. Tunable optical band pass filter when applied different sets of strain on the cross section area of MMF

This figure brief in the table 2.

\begin{tabular}{|c|c|c|c|c|}
\hline $\operatorname{Strain}(\mathbf{m} \varepsilon)$ & Central wavelength(nm) & FWHM(pm) & $A_{d B}=20 \log P_{0}$ & $\Delta \varphi(r a d) \times 10^{-11}$ \\
\hline $\mathbf{0}$ & 1545.726 & 197.529 & 21.66 & 0 \\
\hline 0.09 & 1545.736 & 216.389 & 21.51 & .236 \\
\hline 0.24 & 1545.750 & 233.607 & 20.98 & .261 \\
\hline 0.31 & 1545.764 & 238.081 & 20.83 & .059 \\
\hline
\end{tabular}

Table 2. Micro strain affected on the optical properties of band pass filter

It can be seen from this table, when there is no strain i.e.(zero strain) is applied the $\lambda=1545.726 \mathrm{~nm}, \mathrm{FWHM}=197.529 \mathrm{pm}$, gain $=$ $21.66 \mathrm{~dB}$ and zero phase shift, when strain is applied with $0.09, \lambda=1545.736 \mathrm{~nm}, \mathrm{FWHM}=216.389 \mathrm{pm}$, gain $=21.51 \mathrm{~dB}$ and $(0.236)$ $\mathrm{rad}$, and when applied strain with values $(0.24 \& 0.31)$ we can be seen that the $\lambda$, FWHM are increased and the gain is decreased while the phase shift increase when the length of the multimode fiber is increased, so we can be discussion results in generally, when we applied strain on the second arm of MM- MZI, when this strain is increased leads to $\lambda$ and FWHM are increased gain is decreased and the phase shift increased. 
- Comparison between the simulated and practical results can be observed in these figures.
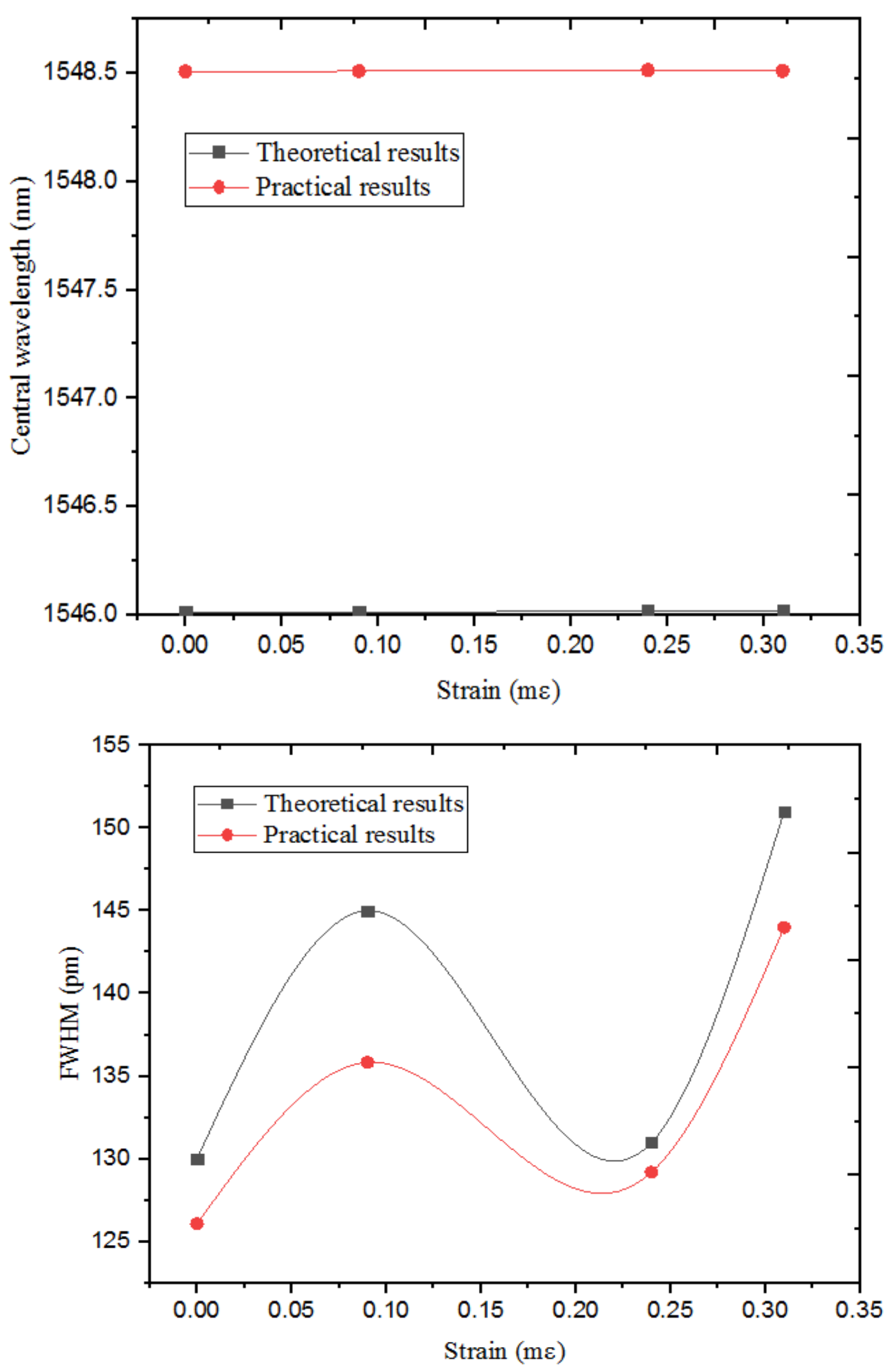


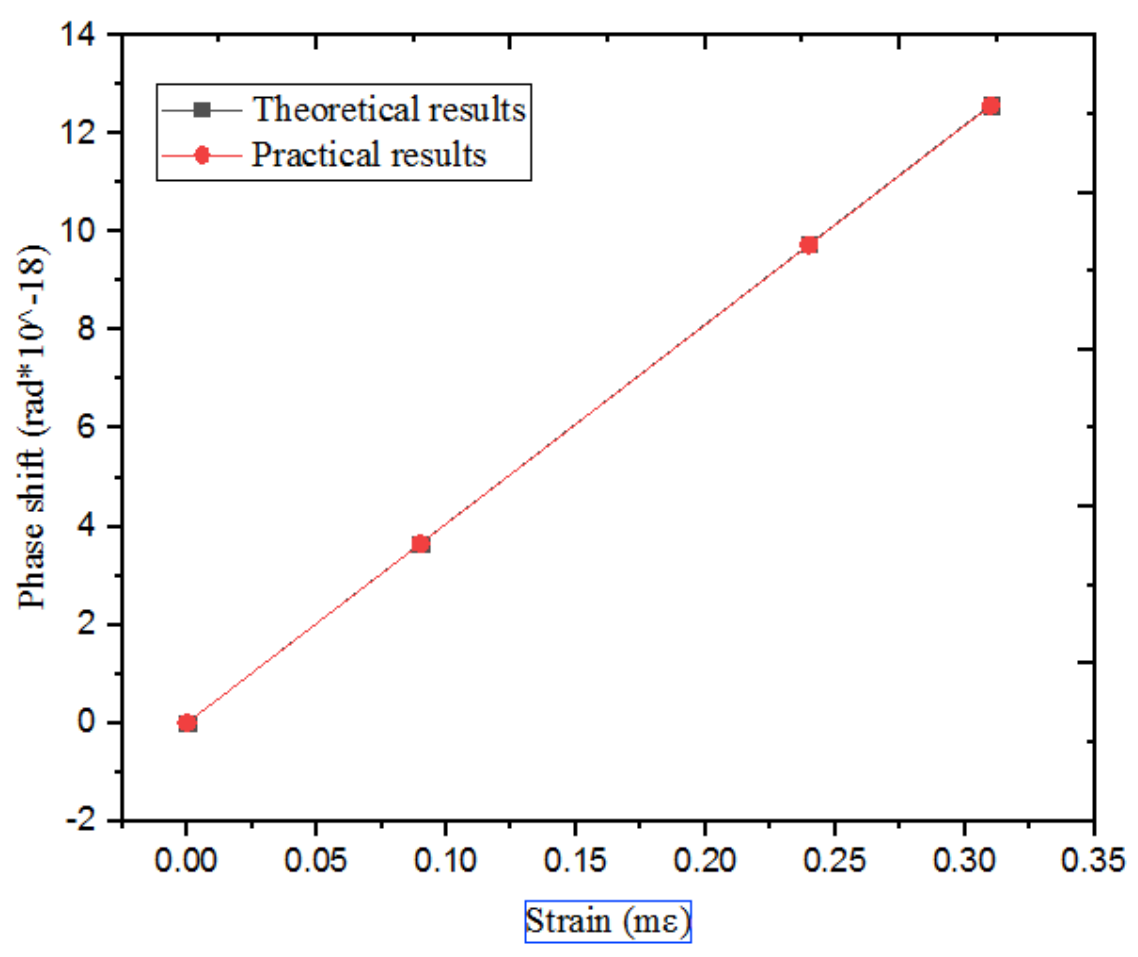

Figure 7. Comparison between the practical and theoretical works with central wavelength, FWHM and phase shift results when applied different sets of strain effect on the cross section area of MMF on the two arms MM-MZI

\section{Conclusion}

Band pass filter and phase modulator was based on MM- MZI, illuminated with a CWLASER source and a fiber length used as dispersive element. When applied Mechanical effect which is micro strain on the second arm of MM-MZI i.e. (effected arm) the length of the fiber was changed can be concluded from the results multimode fiber is not a good band pass filter because of the excitation of higher order modes which effected on the quality of propagating fundamental mode via the core of fiber, but it was a good phase modulator because of the evanesce field. It observed from that, Practical results different from theoretical results because the practical results implemented at laboratory, the humidity in laboratory is large in which temperature up to $45 \mathrm{C}^{\circ}$ and the optical fibers very sensitive to temperature, so changed the refractive index of these fibers. Also the presence of dust in FC connector of the MMF and in the laser pointer and these leads us to some differences between theoretical and practical results.

\section{References}

[1] Escuela. (2004/2005). Fundamental concepts in filter design.

[2] Tai, Yi.,C., Grivas, C., Wilkinson, J. S. (2004). UV photosensitivity in a Ta/sub 2/O/sub 5/ rib waveguide Mach-Zehnder interferometer, IEEE Photonics Technology Letters, 16 (6) 1522 - 1524, 24 (May). 